\title{
AVALIAÇÃO DA SUPERELASTICIDADE EM LIGAS DE NITI RICAS EM NI COM PRÉ-CICLAGEM TÉRMICA VIA ANÁLISES DE ULTRAMICRODUREZA INSTRUMENTADA*
}

\author{
Rodolfo da Silva Teixeira ${ }^{1}$ \\ Saulo Brinco Diniz ${ }^{2}$ \\ Carolline Serafim da Silva ${ }^{3}$ \\ Mônica Aline Magalhães Gurge/ ${ }^{4}$ \\ Andersan dos Santos Paula ${ }^{5}$ \\ Patrícia Freitas Rodrigues ${ }^{6}$ \\ Francisco Manuel Braz Fernandes ${ }^{7}$
}

\section{Resumo}

As ligas de NiTi ricas em Ni tendem a exibir o efeito superelástico a temperatura ambiente. As amostras em estudo foram submetidas a tratamento térmico e mecânico a fim de avaliar o comportamento superelástico destas. Foram realizados ensaios a temperatura ambiente difração de Raios-X (DRX) para identificar as fases presentes e avaliaram-se as propriedades mecânicas via ensaios de ultramicrodureza instrumentada com pré-ciclagem térmica (aquecimento e resfriamento). Os resultados de ultramicrodureza instrumentada exibiram bons indicativos quanto ao uso prévio desta ferramenta para análises do comportamento superelástico destas ligas.

Palavras-chave: Ligas de NiTi ricas em Ni; Superelasticidade; Ultramicrodureza Instrumentada.

\section{EVALUATION OF SUPERELASTICITY IN NI-RICH NITI ALLOYS WITH THERMAL PRE-CYCLING BY INSTRUMENTED ULTRAMICROHARDNESS ANALYSIS}

\begin{abstract}
$\mathrm{Ni}$-rich $\mathrm{NiTi}$ alloys tend to exhibit the superelastic effect at room temperature. The samples under study were submitted to thermal and mechanical treatment in order to evaluate their superelastic behavior. Some experiments are conducted at room temperature associated to X-ray diffraction to identify the present phases and the mechanical properties were evaluated by means of instrumented ultramicrohardness analysis with thermal pre-cycling (heating and cooling). The results of instrumented ultramicrohardness showed good indications regarding the previous use of this tool for analysis of the superelastic behavior of these alloys.
\end{abstract}

Keywords: Ni-rich NiTi Alloys; Superelasticity; Instrumented Ultramicrohardness.

1 Engo Metalurgista, M.Sc., Doutorando em Ciência dos Materiais, PGCM/SE-4, IME, Rio de Janeiro, RJ, Brasil.

2 Engo Metalurgista, M.Sc., Doutorando em Ciência dos Materiais, PGCM/SE-4, IME, Rio de Janeiro, RJ, Brasil; Professor DI - 1, CEFET - Angra dos Reis, Angra dos Reis, RJ, Brasil.

3 Enga Metalurgista, M.Sc., Analista da Garantia da Qualidade Jr., Galvasud, Porto Real, RJ, Brasil.

4 Enga Metalurgista, M.Sc., Doutorando em Ciência dos Materiais, PGCM/SE-4, IME, Rio de Janeiro, RJ, Brasil.

5 Enga Metalurgista, M.Sc. D.Sc., Professora Adjunta, PGCM/SE-4, IME, Rio de Janeiro, RJ, Brasil.

6 Química Industrial, M.Sc., Doutoranda em Ciência dos Materiais, CENIMAT/i3N, FCT - UNL, Caparica, Lisboa, Portugal.

7 Engo Mecânico, M.Sc., D.Sc., Professor Associado, CENIMAT/i3N, FCT-UNL, Caparica, Lisboa, Portugal. 


\section{INTRODUÇÃO}

Diversas pesquisas vêm sendo realizadas recentemente sobre materiais inteligentes, em meio a esta classe de materiais, um de grande destaque tanto acadêmico, quanto comercial são as ligas de Níquel-Titânio (NiTi) de transformações martensíticas termoelásticas, nas quais provém os efeitos de memória de forma (EMF) e superelasticidade (SE). Essas ligas possuem grande interesse devido aliar propriedades de resistência a corrosão, fadiga e biocompatibilidade. Devido as características citadas, além dos EMF e SE, tais ligas apresentam propriedades atrativas para seu desenvolvimento tecnológico, possibilitando diversas aplicações na engenharia (fabricação de microelétrodos de filmes finos, dispositivos elétricos, etc.) e na medicina/odontologia (fios guias cardiovasculares, fios ortodônticos e implantes de placas ósseas [1-5].

A fabricação destas ligas em escala industrial é algo desafiador, devido à dificuldade de deformação, em função da temperatura, a reatividade com oxigênio e carbono devido a presença do Ti que resulta na formação de carbetos e óxidos (TiC e $\mathrm{Ti}_{4} \mathrm{Ni}_{2} \mathrm{O}$ ), o que faz com que seu custo de processamento seja alto pelo uso de equipamentos especiais para elaboração/fusão, perda de material pela oxidação $\left(\mathrm{Ti}_{2} \mathrm{O}\right)$ superficial e formação de trincas durante o processamento termomecânico, usinagem e corte. De acordo com a literatura desempenhos superiores do EMF e SE podem ser obtidos por tratamentos termomecânicos, como deformação a frio seguida de envelhecimento. A deformação a frio promove uma melhora na superelasticidade com 0 aumento da tensão crítica para deslizamento de discordância em relação a transformação de fase induzida por tensão e a demaclagem da martensita (reorientação de suas variantes) [6].

Uma definição simplificada do EMF (Figura 1): é a "memorização" de uma determinada forma prosseguida por uma deformação do material no campo de estabilidade da fase martensítica, seguida por um aquecimento da liga, até o campo de estabilidade da fase mãe, que proporciona o retorno à forma "memorizada". Isto é possível quando um material é deformado, sem auxílio do mecanismo de deslizamento de discordâncias, a uma temperatura abaixo do fim da transformação martensítica $\left(\mathrm{M}_{\mathrm{f}}\right)$, e conserva esta deformação após a remoção da carga e ao ser aquecido, à uma temperatura acima daquela que proporcionará a completa reversão a fase mãe $\left(A_{f}\right)$, readquiri a forma original tanto macro como microestrutural/textural [7].

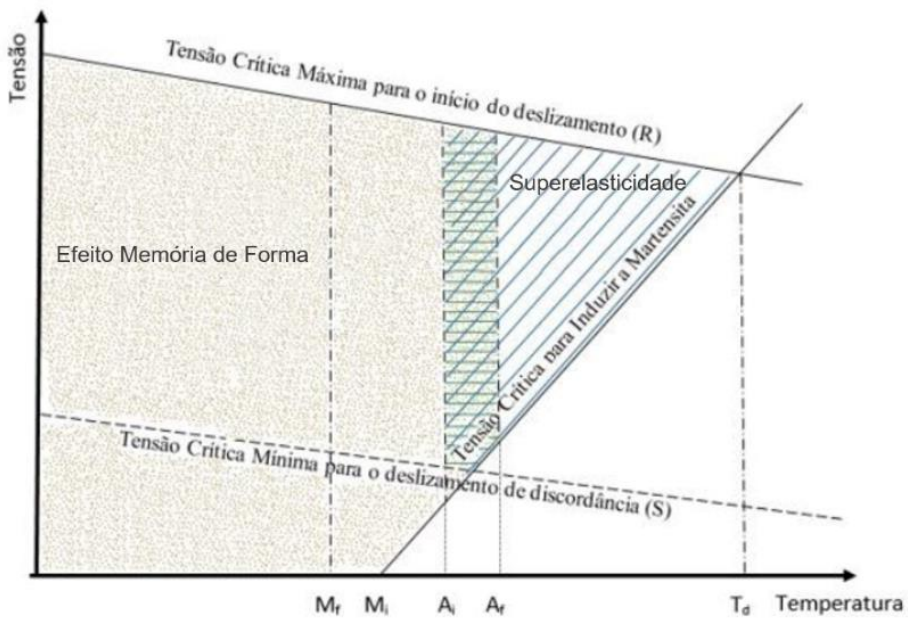

Figura 1. Diagrama esquemático representando a região de efeito de memória de forma e superelasticidade em coordenadas de temperatura-tensão (Adaptado de [7]). 
A SE (Figura 1 - região 2) ocorre no campo da fase mãe $(A)$, dá-se ao deformar um material em uma condição isotérmica, em um intervalo de temperatura acima e próximo a $A_{f}$, a austenita se converte em martensita. Removendo a solicitação imposta, o material retorna a forma e fase original [7].

Com a finalidade de um maior entendimento em relação as análises realizadas neste trabalho, deve-se observar os resultados encontrados no trabalho de Gall et al. [8] sobre nanoindentação instrumentada em ligas de NiTi ricas em Ni (Ti-50,9\%at. Ni), onde indicaram que este ensaio, a longo prazo, pode ser uma opção para identificação das fases presentes apenas com os resultados obtidos via nanoindentação. Neste trabalho, as amostras foram solubilizadas $1000^{\circ} \mathrm{C}$ por $24 \mathrm{~h}$ e envelhecidas nas seguintes temperaturas por $1 \mathrm{~h}: 350,400,450,500$ e $550^{\circ} \mathrm{C}$ e carga máxima de $1000 \mathrm{mN}$. Visto que o envelhecimento pode favorecer a estabilização de fases martensíticas a temperatura ambiente, as amostras foram submetidas ao um aquecimento prévio por alguns minutos a $77^{\circ} \mathrm{C}$ de maneira a assegurar a presença de fase B2 a temperatura ambiente após o resfriamento. A interpretação dos dados de nanoindentação pelos autores ocorreu da seguinte maneira (Figura 3): i) durante o carregamento: no campo martensítico, a reorientação das variantes de martensita; e no campo austenítico, à transformação induzida por tensão seguida de reorientação das variantes de martensita gerada; caso a deformação exceda um limite da reorientação das variantes da martensita, pode ocorrer movimentação de discordâncias. ii) no descarregamento: se tiver a presença de austenita prévia e essa não estiver encruada na região do ensaio, possibilitará uma transformação reversa além da relaxação da tensão devido a deformação elástica.

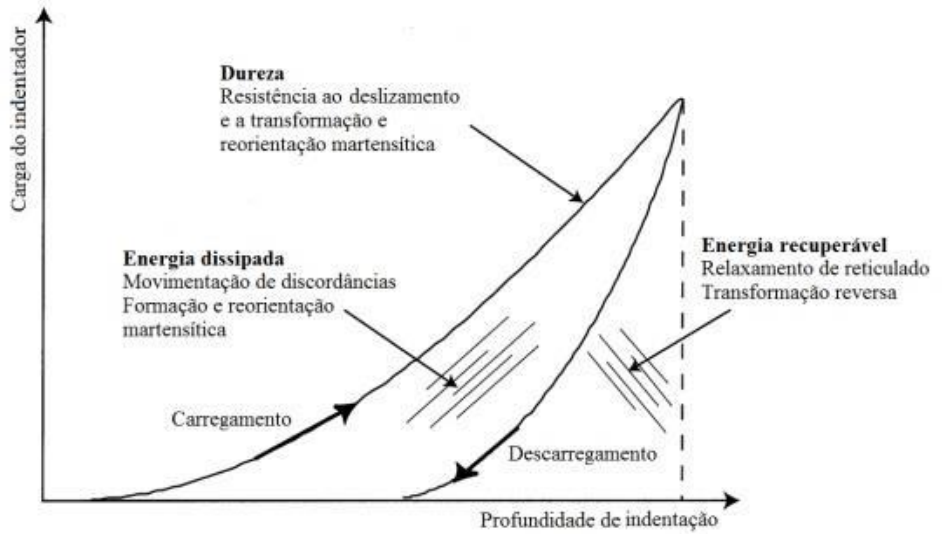

Figura 3. Diagrama dos diferentes mecanismos microestruturais associados a uma curva de nanoindentação em uma liga NiTi (Adaptado de [8]).

Tendo em vista condições estruturais selecionadas de uma determinada sequência de processamento termomecânico (da laminação a quente, passando pela solubilização até a laminação a frio) em uma Liga de $\mathrm{NiTi}$ rica em $\mathrm{Ni}$, na forma de chapa, candidata a exibir superelasticidade. O objetivo do presente trabalho foi o de avaliar a sistemática de pré-aquecimento (em água fervente) ou pré-resfriamento (em nitrogênio) antes de realizar medidas a temperatura ambiente, neste tipo de material, com auxílio das técnicas de Difração de Raios-X (DRX) e ultramicrodureza instrumentada.

Como objetivo secundário confrontar os resultados de dureza dinâmica com medidas de dureza Vickers convencional, obtidas através de medidas em um Ultramicrodurômetro Instrumentado com indentador Vickers, de uma liga de NiTi rica 
em $\mathrm{Ni}$, produzida em escala laboratorial, nas condições selecionadas para o estudo, de modo a verificar evidências de superelasticidade nas mesmas.

\section{MATERIAL E MÉTODOS}

O material em estudo consiste de uma chapa laminada a quente de uma liga de NiTi rica em $\mathrm{Ni}(55 \% \mathrm{Ti}, 0,05 \% \mathrm{C}, 0,084 \% \mathrm{O}$ em peso $(\mathrm{p} / \mathrm{p}))$, elaborada pelo professor e pesquisador Dr. Jorge Otubo do Instituto Tecnológica da Aeronáutica (ITA), São José dos Campos / SP, Brasil. Esta foi fundida em um forno de indução a vácuo (Vaccum Induced Melting Arc -VIM) com cadinho de grafite, onde obteve-se um lingote cilíndrico de $20 \mathrm{~kg}$, do qual extraiu-se uma fatia na forma de disco com 18 $\mathrm{mm}$ de espessura. Esta fatia do lingote submetida ao seguinte tratamento termomecânico, onde foi inicialmente aquecida até $850^{\circ} \mathrm{C} / 1 \mathrm{~h}$ em seguida laminada a quente em múltiplos passes (sem reaquecimento entre estes) até atingir a espessura objetivada $(1,6 \mathrm{~mm})$. Após estas etapas as amostras do material laminado a quente foram solubilizadas a $850^{\circ} \mathrm{C} / 1 \mathrm{~h}$ e resfriada em água a temperatura ambiente. Um grupo de amostra após o tratamento de solubilização foi submetido a uma laminação a temperatura ambiente a fim de reduzir a espessura em $18 \%$, de acordo com o diagrama esquemático apresentado na Figura 4 [9].

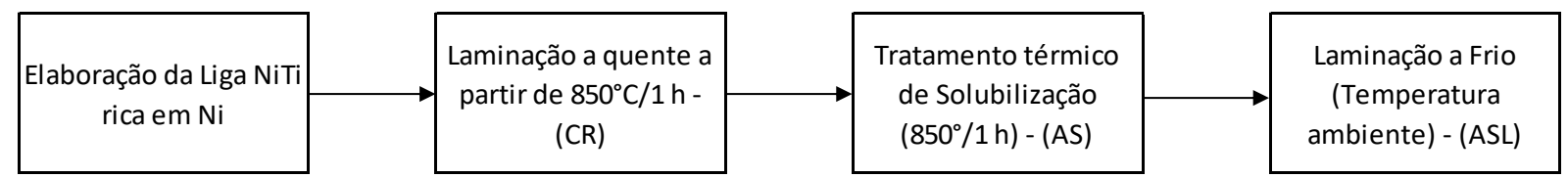

Figura 4. Diagrama esquemático do processamento da amostra.

Foram realizadas análises de difração de Raios-X (DRX) e ultramicrodureza instrumentada.

Para as análises de difração de raios- $X$ as amostras foram lixadas nas seguintes granulometrias: 220, 320, 500, 800, 1200, 2500 e 4000 mesh. Em seguida estas foram decapadas. As medidas de DRX, a temperatura ambiente, foram realizadas no laboratório de Difração de Raios-X do IME utilizando o difratômetro de Raios-X, modelo X'PERT PRO MPD da PANalytical, com anodo de $\mathrm{Cu} \mathrm{K}_{\alpha}$, filtro de níquel, tensão do tubo de $45 \mathrm{kV}$, corrente de $40 \mathrm{~mA}$, com acoplamento Theta/2Theta, passo de $0,050^{\circ}$ de 2 Theta, com velocidade de varredura de 2,3 segundos para o intervalo entre 30 a $110^{\circ}$ de 2 Theta.

Nas análises de ultramicrodureza as amostras também foram lixadas nas mesmas condições que as da análise de DRX, porém foram submetidas a um polimento eletrolítico com as seguintes condições: solução com $20 \%$ de ácido sulfúrico e $80 \%$ de metanol a temperatura ambiente e com tensão (15 ou $30 \mathrm{~V}$ ) e tempo variando entre 3 a 10 segundos. Em cada amostra foram realizados 10 ensaios ao longo de 1/2 espessura na seção transversal associada a direção de laminação, nas condições pré-aquecidas e pré-resfriadas (conforme procedimento descrito abaixo). A carga máxima adotada nas condições de carga e descarga, sem tempo de permanência na carga máxima ou mínima foi de 20 gf $(196,33 \mathrm{mN})$, com taxa de carregamento/descarregamento de $70,0670 \mathrm{mN} / \mathrm{s}$.

Tanto para as análises de DRX como de ultramicrodureza os ensaios foram conduzidos a temperatura ambiente (com temperatura controlada em $20^{\circ} \mathrm{C}$ ), porém para uma mesma amostra duas medidas foram feitas:

- num primeiro momento esta foi pré-aquecida em água e mantida até a homogeneização da temperatura, após $X$ minutos de permanência estas foram 
resfriadas ao ar na condição climatizada dos respectivos laboratórios onde estão instalados os equipamentos e realizadas as medidas.

- num segundo momento essa mesma amostra depois de ensaiada na condição anterior de pré-aquecimento, foi submetida a um resfriamento em nitrogênio líquido por tempo suficiente até a homogeneização da temperatura (5 min) e removida para aquecer naturalmente até a temperatura ambiente, em seguida ensaiada para fins de comparação.

\section{RESULTADOS E DISCUSSÃO}

$\mathrm{Na}$ Figura 5 são apresentadas as análises de difração de raios-X. Na amostra laminada a quente a partir de $850^{\circ} \mathrm{C} / 1 \mathrm{~h}$ (Figura 5(a)) constatou-se que o material exibe somente picos alargados da fase B2, a temperatura ambiente, para ambas condições prévias (pré-aquecida e pré-resfriada), onde os difratogramas são muito semelhantes. Estes resultados estão de acordo com análises observadas na literatura (10), reforçando assim a hipótese que a laminação a quente adotada (em função do decréscimo progressivo da temperatura de deformação a cada passe) promoveu um encruamento significativo da austenita que resultou num incremento considerável da tensão residual do material. Ou seja, o estado estrutural da austenita nesta condição acarretou na supressão das transformações de fase condição pré-resfriada (em nitrogênio líquido) e pré-aquecida (100드 referentes a água fervente) das análises de DRX efetuadas nas amostras posteriormente estabilizadas a temperatura ambiente. Na amostra solubilizada $850^{\circ} \mathrm{C} / 1 \mathrm{~h}$ (Figura 5(b)) observou-se que os picos da fase B2 e fase-R, a temperatura ambiente, tanto para condição prévia de aquecimento e resfriamento. Os difratogramas são muito semelhantes, porém na condição pré-aquecida apresentou uma redução da

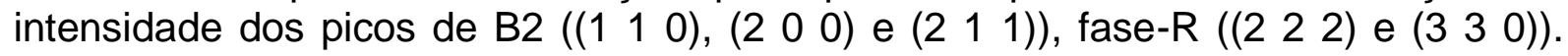
Quando confrontados os resultados da literatura (10), estes estão de acordo. Numa amostra totalmente austenítica (AS), porém com nível de tensão de residual inferior (evidenciado pelo estreitamento dos picos de B2) quando comparada a amostra laminada a quente em estudo (CR), considerando-se que esta foi submetida ao tratamento de solubilização a $850^{\circ} \mathrm{C}$. Na amostra com laminação a temperatura ambiente (ASL), após a solubilização, (Figura 3(c)) verificou-se que o material exibe somente picos de difração alargados da fase B2, em ambas condições prévias. Os difratogramas são muito semelhantes, no entanto este alargamento muito mais acentuado do que aquele evidenciado para a amostra laminada a quente (CR) (Figura 5(a)). 

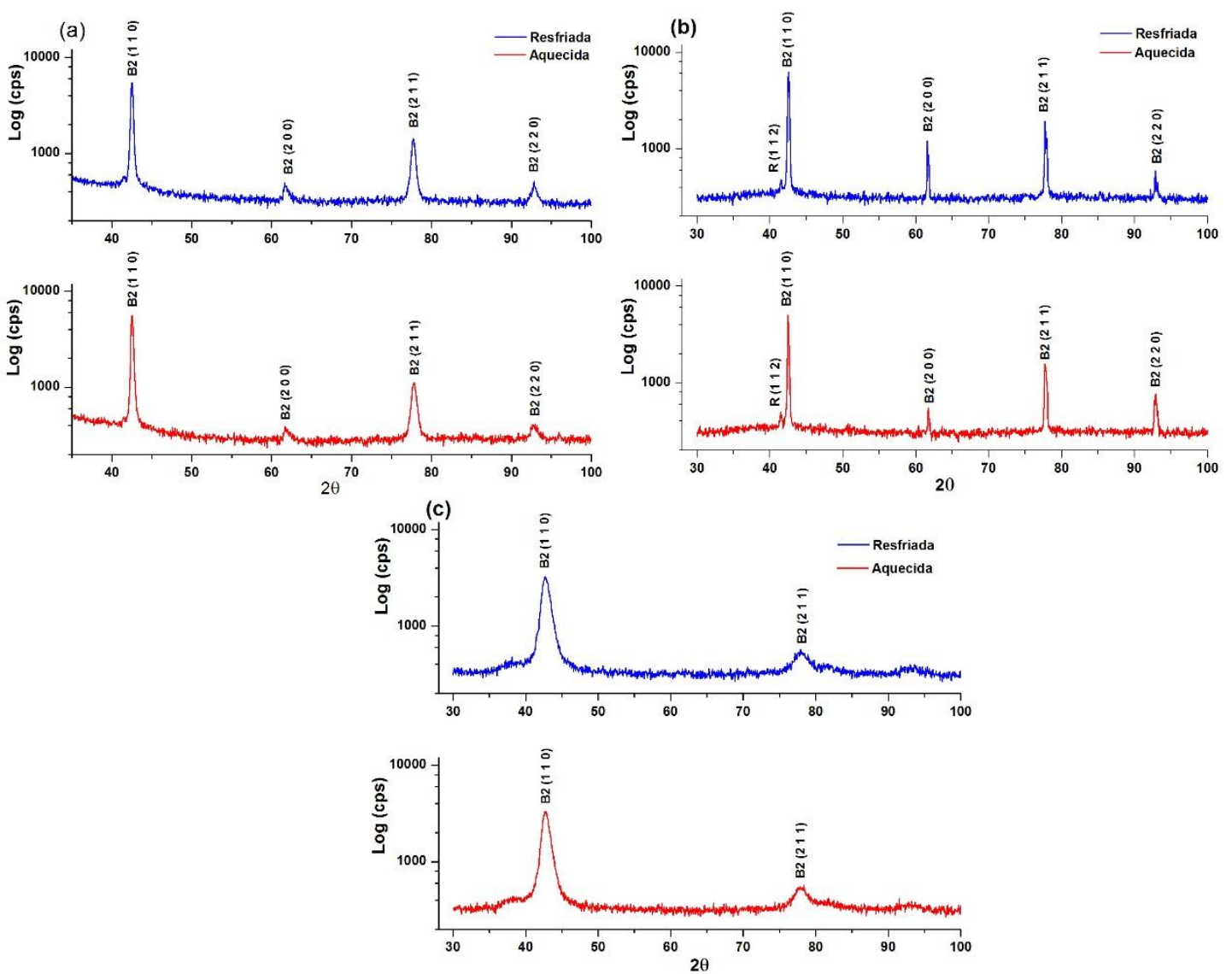

Figura 5. Difratogramas, análise a temperatura ambiente, confronto após aquecimento e pré-

resfriamento: (a) chapa laminada a quente a partir de $850^{\circ} \mathrm{C} / 1 \mathrm{~h}(\mathrm{CR})$; (b) chapa solubilizada $850^{\circ} \mathrm{C} / 1 \mathrm{~h}$ (AS); (c) chapa solubilizada $850^{\circ} \mathrm{C} / 60 \mathrm{~min}$ com posterior laminação a temperatura ambiente com $18 \%$ de redução na espessura (ASL).

$\mathrm{Na}$ Figura 6 são apresentados os gráficos com os resultados das análises de ultramicrodureza instrumentada. Os valores de dureza Vickers convencional (Figura 6(a)) foi obtido através da Equação 1,

$$
H V=\frac{189.10 x F}{L^{2}}
$$

Onde,

F é a força máxima $(\mathrm{mN})$;

$L$ é a média das diagonais da endentação $(\mu \mathrm{m}), L=\frac{\left(L_{1}+L_{2}\right)}{2}$.

O maior valor de HV foi para amostra ASL, seguida da amostra CR. Possivelmente está associado a presença de encruamento no material. No caso da amostra ASL a laminação a temperatura ambiente e na amostra CR as etapas finais de laminação a quente provavelmente promoveram a retenção de encruamento na estrutura da fase B2, já que a redução ocorreu em temperaturas abaixo daquela que garante a ocorrência de fenômenos de recristalização dinâmica ou estática no material. A dureza dinâmica elasto-plástica (DHV-1) foi obtida através da Equação 2,

$$
D H V-1=\frac{a . F}{(h m a x)^{2}}
$$


Onde,

a é uma constante que depende da forma do indentador $(3,8584$ para um indentador Vickers);

F é a força máxima $(\mathrm{mN})$;

$h_{\max }$ é a profundidade máxima de indentação.

A fim de comparação, a dureza DHV-1 pode ser comparada a dureza vickers. $O$ comportamento global desta (Figura 4(b)) é semelhante à dureza vickers (Figura 6(a)). Porém, exibiu um incremento para a amostra CR.

Já a dureza dinâmica plástica (DHV-2) foi obtida através da Equação 3,

Onde,

$$
D H V-2=\frac{a \cdot F}{(h r)^{2}}
$$

a é uma constante que depende da forma do indentador (3,8584 para um indentador Vickers);

F é a força máxima $(\mathrm{mN})$;

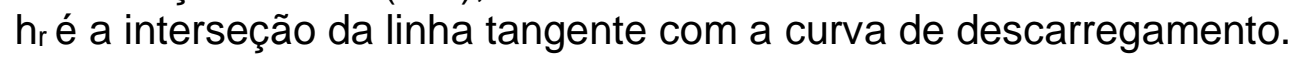

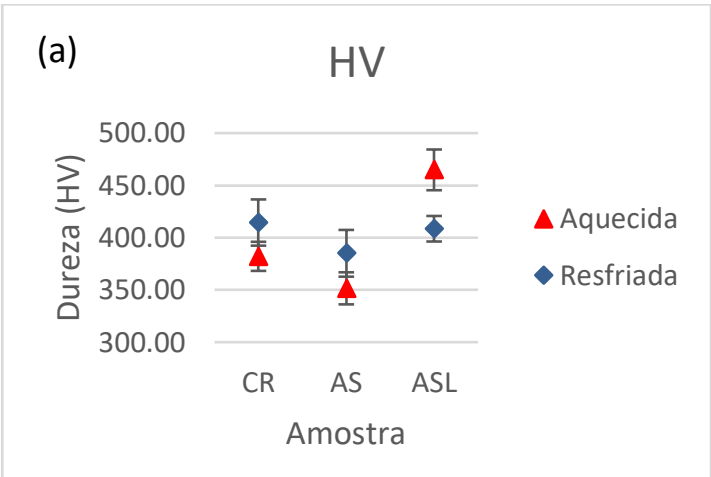

(b)

DHV-1

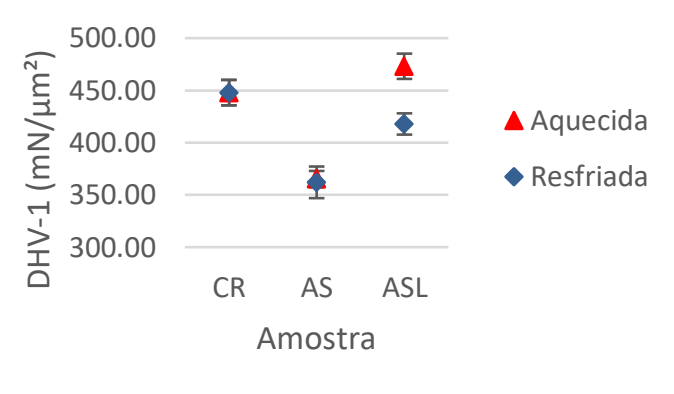

(c)

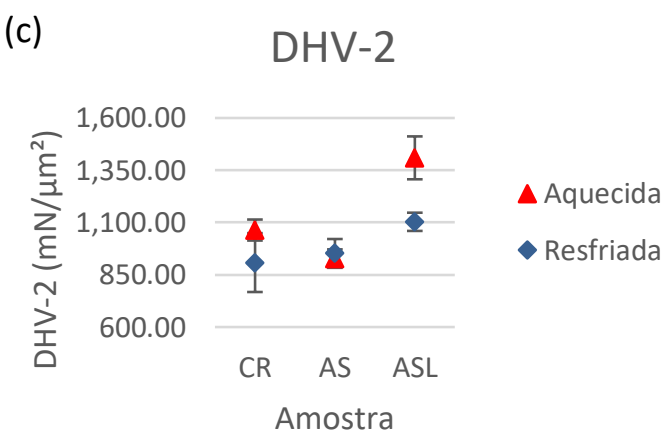

Figura 6. Análises de ultramicrodureza instrumentada das amostras nas condições em estudo, confronto após aquecimento e pré-resfriamento: (a) Dureza HV, (b) Dureza DHV-1 e (c) Dureza DHV2.

A dureza dinâmica plástica (DHV-2) (Figura 6(c)) exibiu valores superiores para a amostra ASL. Um dos motivos para este comportamento está associado às inclinações das curvas, onde para esta amostra foram maiores quando comparada as demais. Também é possível que esteja associado à laminação previa, está pode ter promovido um incremento de fase- $\mathrm{R}$ no material. De acordo com a literatura a presença de fase- $\mathrm{R}$ no material promove melhor efeito superelástico.

Como continuidade dos estudos relativos as transformações de fases presentes nas amostras da liga em estudo, estão sendo conduzidas análises térmicas via Calorimetria Diferencial de Varredura (DSC) e dilatometria de modo a averiguar a 
ocorrência de transformações de fases, bem como análise de DRX in situ com ciclagem térmica a fim definir as respectivas sequencias de transformações de fases presentes nas condições de processamento analisadas para a liga em estudo.

\section{CONCLUSÃO}

- O pré-processamento de aquecimento (em água em ebulição) / pré-resfriamento (nitrogênio líquido) promoveu diferentes misturas de fase nas amostras em estudo detectadas por meio da análise de difração de raios $X$ realizada à temperatura ambiente;

- As propriedades HV e DHV-1, exibiram valores superiores de dureza na condição pré-resfriada, com exceção da amostra ASL. É possível que esse comportamento esteja associado ao resfriamento em $\mathrm{Ni}$ líquido ao qual as amostras foram submetidas, onde a temperatura do nitrogênio líquido $\left(-197^{\circ} \mathrm{C}\right)$, pode ter sido suficiente para induzir a formação de alguma pequena fração de fase $R$ em conjunto com uma fração ainda muito significativa de fase B2.

- Detectou-se a presença de fase-R na amostra solubilizada, onde era esperada apenas a presença de fase austenítica (B2).

- Somente com as análises de dureza Vickers não foi possível inferir sobre o efeito superelástico deste material;

- Quanto às propriedades de dureza dinâmica elasto-plástica (DHV-1) pode ser uma análise alterativa para avaliação da dureza do material, tendo em vista a semelhança dos resultados com HV.

- Os resultados de dureza dinâmica plástica (DHV-2) foram mais efetivo no comportamento superelástico para amostra ASL, tanto pré-aquecida como resfriada;

- A análise de ultramicrodureza instrumentada pode ser uma ferramenta para uma análise prévia do comportamento superelástico nessas ligas. Porém, tão somente está é suficiente para que se possa inferir quanto ao efeito superelástico. É necessária a realização de ensaios mecânicos convencionais, tais como testes de tração, flexão, etc.

\section{Agradecimentos}

Os autores agradecem ao professor J. Otubo por doar os materiais de partida, que foi elaborado no Instituto de Tecnologia Aeronáutica (São José dos Campos, SP Brasil).

P.F.R e F.M.B.F reconhecem o financiamento da CENIMAT / i3N. Aos recursos do projeto MIDAS, de fomento europeu, que permitiram que os ensaios de DSC no CENIMAT/i3N fossem conduzidos.

Ao IME pelos recursos para preparação e caracterização microestrutural. Ao Laboratório de Caracterização de Materiais Multiusuários no Instituto de Ciências Exatas (ICEx) da UFF pela disponibilização de uso do ultramicrodurômetro instrumentado. R.S. Teixeira agradece a bolsa de mestrado subsidiada pela CNPq vinculada ao PGCM/SE4-IME.

A.S.P. agracede a bolsa de produtividade à pesquisa (PQ-2) do CNPq (Processo 307798/2015-1).

\section{REFERÊNCIAS}


1 Paula AS. Tratamentos termomecânicos de ligas do sistema $\mathrm{Ni}-\mathrm{Ti}$ - caracterização estrutural e optimização das propriedades associadas ao efeito de memória de forma [tese de Doutorado]. Lisboa: Universidade Nova de Lisboa; 2006.

2 Tabib-Azar M, Sutapun B, Huff M. Applications of TiNi thin film shape memory alloys in micro-opto-electro-mechanical systems. Sensors and Actuators, A. 1999; 77 (1): 34-38.

3 Jardine AP et al. Shape memory wire improves sealing of aircraft doors, Patent number: US6009669, 2000.

4 Duerig T, Pelton A, Stöckel D. An overview of nitinol medical applications. Materials Science and Engineering: A. 1999; 273-275:149-160.

5 Castilho WS, Silva EP. Algumas aplicações das ligas com memória de forma shape memory alloys - SMA. In: Congresso Sinergia, v. 12, n. 1,2011, 99-108.

6 Fatemeh Khaleghi, Jafar Khalil-Allafi, Vahid Abbasi-Chianeh, Soheil Noori. Effect of short-time annealing treatment on the superelastic behavior of cold drawn Ni-rich NiTi shape memory wires. Journal of Alloys and Compounds. 2013; 554: 32-38.

7 Otsuka K, Wayman CM. Shape memory materials. Cambridge: Cambridge University Press; 1998.

8 Gall K. †, Juntunen K., Maier HJ, Sehitoglu H., Chumlyakov YI. Instrumented microindentation of NiTi shape-memory alloys. Acta Materialia. 2001; 49(16): 3205-3217.

9 Oliveira GCM. Caracterização microestrutural e mecânica da liga NiTi com emf produzida em forno de indução a vácuo [dissertação de mestrado]. São José dos Campos: Instituto Tecnológico de Aeronáutica; 2010.

10 Rodrigues PF. Evolução estrutural e das transformações de fase em uma liga de NiTi equiatômica laminada a quente submetida a tratamentos térmicos de solubilização e envelhecimento em tempos curtos [dissertação de mestrado]. Volta Redonda: Universidade Federal Fluminense; 2012. 